\title{
Dendrometria de espécies nativas em plantios homogêneos no Estado de Roraima - Andiroba (Carapa guianensis Aubl), Castanha-do-Brasil (Bertbolletia excelsa Bonpl.), Ipê-roxo (Tabebuia avellanedae Lorentz ex Griseb) e Jatobá (Hymenaea courbaril L.)
}

\author{
Helio TONINI ${ }^{1}$; Marcelo Francia ARCO-VERDE ${ }^{1}$; Sergio Pedreira Pereira de SÁ ${ }^{1}$
}

\begin{abstract}
RESUMO
Estudou-se o crescimento e a seleção de equações para quatro espécies florestais nativas visando identificar espécies promissoras para o plantio em programas de reflorestamentos e em sistemas agroflorestais no Estado de Roraima. O crescimento da andiroba (Carapa guianensis Aubl.), da castanha-do-brasil (Bertholletia excelsa Bonpl.), do ipê-roxo (Tabebuia avellanedae Lorentz ex Griseb.) e do jatobá (Hymenaea courbaril L.), aos sete anos de idade, mostrou-se promissor atingindo incrementos médios anuais em volume comercial de 6.3; 14.6; 6.0 e $2.3 \mathrm{~m}^{3} \cdot \mathrm{ha}^{-1}$.ano ${ }^{-1}$, respectivamente. Em relação ao crescimento em diâmetro, todas as espécies apresentaram incrementos médios anuais em diâmetro maiores do que $1 \mathrm{~cm}$, sendo superiores aos observados para árvores crescendo em florestas naturais. A análise estatística, indicou a equação hipsométrica de Prodan como a de melhor ajuste para estimar a altura em função do diâmetro para as quatro espécies analisadas. No entanto, a análise gráfica indicou que a forma da curva altura/diâmetro variou com a espécie sendo necessário o ajuste em separado. $O$ ajuste de equações de volume comercial com casca e fator de forma comercial mostraram ser necessário o ajuste de diferentes equações em função da espécie. A análise gráfica das curvas de volume comercial e fator de forma indicaram que as espécies diferiram em ambos os parâmetros, indicando que a utilização de um fator de forma médio para todas as espécies deve ser evitado, como forma de aumentar a precisão nas estimativas volumétricas.
\end{abstract}

PALAVRAS -CHAVE

Brasil, Amazônia, volume comercial, fator de forma

\section{Dendrometry of native species in bomogeneous stands in the Roraima State - andiroba (Carapa guianensis Aubl), castanba-do-brasil (Bertholletia excelsa Bonpl), ipêe-roxo (Tabebuia avellanedae Lorentz ex Griseb) and jatobá (Hymenaea courbaril L.)}

\begin{abstract}
The growth and selection of equations for fournative forest species was studied aiming to identify promising speciesfor homogeneous stands, and agroflorestry systems in the Roraima state. The growth of andiroba (Carapa guianensis Aubl.), castanha-do-brasil (Bertholletia excelsa Bonpl.), ipê-roxo (Tabebuia avellanedae Lorentzex Griseb) and jatobá (Hymenaea courbaril L.) to the seven years of age, revealed to be promising, reaching mean annual increments in merchantable volume of 6.3; 14.6; 6.0 and $2.3 \mathrm{~m}^{3} . \mathrm{ha}^{1}$ year ${ }^{1}$, respectively. Regarding diameter growth, all the species presented mean annual increments over than $1 \mathrm{~cm}$, being higher than the observed ones for trees growing in natural forests. The statistical analyses indicated the hypsometric Prodan equation as of better adjustment for the analyzed species. However, the graphical analysis indicated that the shape of the b/d curve varied with the species. The fit of merchantable volume and commercial form factor equations demonstrated to be necessarily different equations for each species. The graphical analysis of the merchantable volume and form factor curves showed that the species differed in both the parameters, indicating that the use of an average form factor for all the species must be avoided.
\end{abstract}

KEY WORDS

Brazil, Amazônia, merchantable volume, form factor

1 Eng $^{0}$ Florestal, Pesquisador do Cpaf Embrapa Roraima, Br 174, Km 08, Distrito Industrial, CEP 69301-970, Boa Vista/RR.Email: helio@cpafrr.embrapa.br; arcoverd@cpafrr.embrapa.br;sergiosa@cpafrr.embrapa.br 


\section{INTRODUÇÃO}

A taxa de desmatamento e a conseqüente diminuição da cobertura de florestas tropicais na Amazônia têm sido motivo de preocupação mundial. A expansão da fronteira agropecuária, as queimadas e a erosão têm sido apontadas como responsáveis pela degradação de enormes superfícies nesta região.

A substituição da floresta natural pela agricultura e pecuária, em solos cada vez menos estáveis e produtivos, vem acompanhada por um aumento da pobreza e a perda da capacidade produtiva da terra, tanto para cultivos agrícolas como para florestas, exigindo a busca de alternativas capazes de assegurar rendimentos sustentáveis.

Neste contexto, o papel do reflorestamento ou florestamento na proteção dos recursos hídricos e edáficos, na recuperação de áreas degradadas, na alta demanda por mão de obra e pela expectativa futura de aumentos substanciais nos preços dos produtos florestais resultantes do aumento do desmatamento e restrições legais, o torna uma alternativa viável sob os pontos de vista ecológico, econômico e social.

Segundo Wadsworth (2000), o cultivo de árvores exige menos nutrientes e tolera maior acidez e toxidade ao alumínio do que a maioria dos cultivos agrícolas. A vocação florestal das regiões tropicais é uma conseqüência do próprio comportamento dos solos, em que o excesso de precipitação e as temperaturas elevadas durante todo o ano são condições que favorecem o empobrecimento da terra por lixiviação (Thibau, 2000).

Um plantio florestal tem grande importância para o regime hídrico de um local, diminuindo o impacto das gotas de chuva por interceptação, reduzindo a erosão, possibilitando o aumento da infiltração de água no solo. Pode-se estimar que cada centímetro de espessura de serrapilheira é capaz de reter e infiltrar $2 \mathrm{~mm}$ de chuva (Andrae, 1978).

Segundo Thibau (2000), a análise química das plantas, em diversos estudos, mostra que a biomassa ou a matéria vegetal desidratada compõe-se de $44 \%$ de carbono, que é incorporado ou fixado durante a fotossíntese. Florestas jovens em crescimento, ou em regeneração, possuem um índice de área foliar muito superior aos tecidos lenhosos ocasionando alta produtividade primária e elevada fixação de carbono.

Os argumentos econômicos a favor das plantações baseiamse principalmente na disponibilidade de terrenos e na expectativa de escassez futura de madeira. Para Wadsworth (2000), a superioridade de plantações sobre florestas naturais deve-se principalmente à sua maior produtividade de madeira comercial. Estas vantagens são mais evidentes onde a regeneração é pobre e onde as árvores nativas são de utilidade limitada.

Entre os principais critérios para a seleção de espécies para reflorestamento estão a aptidão em relação ao sítio e a elevada produtividade (Lamprecht, 2000). A escolha de espécies é um sério problema em países tropicais e subtropicais, onde o uso de uma espécie em local inadequado pode levar a problemas como: produtividade inferior ao potencial da região; elevada suscetibilidade ao ataque de pragas; inadaptação da espécie e produção de madeira com características inadequadas (Ferreira, 1987).

Os trabalhos dendrométricos, em geral, são relacionados com espécies introduzidas, de rápido crescimento, principalmente os gêneros Pinus e Eucalyptus. As espécies nativas muitas vezes deixam de ser estudadas por não conseguirem despertar interesse equivalente, na maioria das vezes, pela inexistência de informações relativas a sua ecologia, silvicultura e biometria.

Portanto, este trabalho foi realizado com o objetivo de estudar o crescimento de quatro espécies florestais nativas, visando identificar espécies para reflorestamentos homogêneos e sistemas agrosilviculturais no estado de Roraima e selecionar equações para expressar o comportamento das principais variáveis dendrométricas utilizadas nos modelos de crescimento e produção.

\section{MATERIAIS E MÉTODOS}

\section{Características do local}

A partir de 1995 foi iniciado, pela Embrapa Roraima, o plantio de uma coleção de espécies arbóreas nativas e introduzidas, com a finalidade de avaliar preliminarmente espécies potenciais para sistemas agroflorestais e programas de reposição florestal. Aárea experimental localiza-se no Campo Experimental Confiança, no município do Cantá, distante cerca de $90 \mathrm{~km}$ da capital do Estado, Boa Vista.

O clima na região é classificado como do tipo Ami (Köppen), com precipitação média de $1900 \mathrm{~mm}$, com época chuvosa ocorrendo no período de abril a setembro, sendo o mês de junho, o mais chuvoso, representando cerca de 19\% da precipitação total anual.

O solo é classificado como do tipo argissolo, constituído por material mineral que tem como características argila de atividade baixa e horizonte B textural (Embrapa, 1999). As características químicas são apresentadas na Tabela 1.

Tabela 1 - Análise química dos solos, nas profundidades de 0-20 e 20-40 cm, no Campo Experimental Confiança da Embrapa Roraima.

\begin{tabular}{|c|c|c|c|c|c|c|c|}
\hline $\begin{array}{l}\text { Profundidade } \\
(\mathrm{cm})\end{array}$ & $\begin{array}{l}\mathrm{pH} \\
\left(\mathrm{H}_{2} \mathrm{O}\right)\end{array}$ & $\begin{array}{l}\mathrm{Al} \\
\mathrm{cmolc} / \mathrm{dm}^{3}\end{array}$ & $\begin{array}{l}\text { M.O. } \\
\text { g/dm }\end{array}$ & $\begin{array}{l}\mathrm{P} \\
\mathrm{mg} / \mathrm{dm}^{3}\end{array}$ & $\begin{array}{l}\mathrm{K} \\
\mathrm{mg} / \mathrm{dm}^{3}\end{array}$ & $\begin{array}{l}\mathrm{Ca} \\
\mathrm{cmolc} / \mathrm{dm}^{3}\end{array}$ & $\begin{array}{l}\mathrm{Mg} \\
\mathrm{cmolc} / \mathrm{dm}^{3}\end{array}$ \\
\hline $0-20$ & 4,2 & 1,27 & 36,8 & 3,42 & 22,45 & 0 & 0,1 \\
\hline $20-40$ & 4,3 & 1,07 & 20,5 & 0,4 & 7,6 & 0 & 0,05 \\
\hline
\end{tabular}




\section{ACTA \\ AMAZONICA}

DENDROMETRIA DE ESPÉCIES NATIVAS EM PLANTIOS HOMOGÊNEOS NO ESTADO DE RORAIMA ANDIROBA (Carapa guianensis AUBL), CASTANHA-DO-BRASIL (Bertholletia excelsa BONPL.), IPÊROXO (Tabebuia avellanedae LORENTZ EX GRISEB) E JATOBÁ (Hymenaea courbaril L.)

\section{Coleta dos dados}

Os dados para a realização deste estudo foram oriundos da medição de 87 árvores, sendo 19 de Carapa guianensis (andiroba), 21 de Bertholletia excelsa (castanha-do-brasil), 22 de Tabebuia avellanedae (ipê-roxo) e 25 de Hymenaea courbaril (jatobá). As árvores foram plantadas em parcelas de $180 \mathrm{~m}^{2}$ em espaçamento de $2,5 \times 2 \mathrm{~m}$, totalizando 30 plantas

Tabela 2 - Equações de relação hipsométrica ajustadas para Carapa guianensis (andiroba), Bertbolletia excelsa (castanhado-brasil), Tabebuia avellanedae (ipê-roxo) e Hymenaea courbaril (jatobá).

\begin{tabular}{|c|c|}
\hline $\begin{array}{l}N^{0} \text { da } \\
\text { equação }\end{array}$ & Equação \\
\hline 01 & $\frac{1}{\sqrt{h-1,3}}=b_{0}+b_{1} \frac{1}{d}$ \\
\hline 02 & $h-1,3=b_{0}+b_{1} d+b_{2} d^{2}$ \\
\hline 03 & $\frac{1}{h-1,3}=\frac{1}{b_{o}+b_{1} d+b_{2} d^{2}}$ \\
\hline 04 & $h-1,3=b_{0}+b_{1} d$ \\
\hline 05 & $h=b_{0}+b_{1} d$ \\
\hline 06 & $\ln (h-1,3)=b_{0}+b_{1} \frac{1}{d}$ \\
\hline 07 & $\ln (h)=b_{0}+b_{1} \frac{1}{d}$ \\
\hline 08 & $\ln (h)=b_{0}+b_{1} d$ \\
\hline 09 & $\ln (h-1,3)=b_{0}+b_{1} \ln (d)$ \\
\hline 10 & $\ln (h-1,3)=b_{0}+b_{1} \ln (d)+b_{2} \ln ^{2}(d)$ \\
\hline 11 & $\ln (h-1,3)=b_{0}+b_{1} \ln \left(\frac{d}{1+d}\right)$ \\
\hline 12 & $h=b_{0}+b_{1} d+b_{2} d^{2}$ \\
\hline 13 & $h=b_{0}+b_{1} d+b_{2} d^{2}+b_{3} d^{3}$ \\
\hline 14 & $h=b_{0}+b_{1} \frac{1}{d}$ \\
\hline 15 & $\frac{d^{2}}{\sqrt{h-1,3}}=b_{0}+b_{1} d+b_{2} d^{2}$ \\
\hline
\end{tabular}

Fonte: Finger (1992)

Sendo: $\mathrm{h}=$ altura total $(\mathrm{m}) ; \mathrm{d}=$ diâmetro a altura do peito;

$b_{o^{\prime}}, b_{1}, b_{2}$ e $b_{3}=$ coeficientes. por parcela. Os plantios foram feitos por coveamento direto, com a aplicação de $60 \mathrm{~g}$ de fósforo na cova.

Em cada parcela, foram medidos o diâmetro à altura do peito (DAP), tomado a $1,30 \mathrm{~m}$ do solo e a altura total (h). O volume comercial foi obtido pela cubagem rigorosa de todas as árvores em pé, com auxílio de escadas, até o diâmetro limite de $7 \mathrm{~cm}$ com casca. Para o cálculo do volume, utilizou-se a metodologia de Smalian, sendo tomadas os diâmetros com casca nas posições 0,$1 ; 0,7 ; 1,30 \mathrm{~m}$ e a partir deste ponto, de metro em metro até o diâmetro limite.

$O$ fator de forma comercial foi obtido pela razão entre $o$ volume comercial e o volume do cilindro com DAP e altura igual a da árvore, pela seguinte expressão:

$$
f_{c}=\frac{v_{\text {comercial }}}{v_{\text {cilindro }}}
$$

\section{Relação hipsométrica}

Para descrever o crescimento em altura em função do DAP foram testadas, para cada espécie, 15 funções de relação hipsométrica, que são apresentadas na Tabela 2.

Tabela 3 - Equações de volume de simples entrada ajustadas para Carapa guianensis (andiroba), Bertbolletia excelsa (castanha-do-brasil), Tabebuia avellanedae (ipê-roxo) e Hymenaea courbaril (jatobá).

\begin{tabular}{lll}
\hline \hline $\begin{array}{l}\mathbf{N}^{0} \text { da } \\
\text { equação }\end{array}$ & \multicolumn{1}{c}{ Equação } & Autor \\
\hline 01 & $v=b_{o}+b_{1} d$ & $\begin{array}{l}\text { Dissescu- } \\
\text { Stanescu }\end{array}$ \\
02 & $v=b_{o}+b_{1} d^{2}$ & $\begin{array}{l}\text { Hohenald- } \\
\text { Krenn }\end{array}$ \\
03 & $v=b_{o}+b_{1} d+b_{2} d^{2}$ & Berkhout \\
04 & $\ln v=b_{0}+b_{1} \ln d$ & Brenac \\
05 & $\ln v=b_{0}+b_{1} \ln d+b_{2} \frac{1}{d}$ & \\
06 & $v=b_{o}+b_{1} \frac{1}{d}$ & \\
07 & $v=b_{o}+b_{1} \frac{1}{d}+b_{2} d$ & \\
08 & $\ln v=b_{0}+b_{1} \ln d+b_{2} d$ & \\
09 & $\ln v=b_{o}+b_{1} d+b_{2} d^{2}$ & \\
\hline \hline
\end{tabular}

Fonte: Finger (1992)

Sendo: $v$ = volume comercial com casca $\left(\mathrm{m}^{3}\right) ; \mathrm{d}=$ diâmetro $\mathrm{a}$ altura do peito; $\mathrm{b}_{\mathrm{o}^{\prime}}$ $b_{1}, b_{2}=$ coeficientes. 


\section{ACTA AMAZONICA}

Tabela 4 - Equações de volume de dupla entrada ajustadas para Carapa guianensis (andiroba), Bertbolletia excelsa (castanha-dobrasil), Tabebuia avellanedae (ipê-roxo) e Hymenaea courbaril (jatobá).

\begin{tabular}{lll}
\hline \hline $\begin{array}{l}\mathbf{N}^{0} \mathrm{da} \\
\text { equação }\end{array}$ & Equação & Autor \\
\hline 01 & $v=b_{o}+b_{1} d^{2} h$ & Spurr \\
02 & $v=b_{o}+b_{1} d^{2}+b_{2} d^{2} h+b_{3} h$ & Stoate \\
03 & $v=b_{o}+b_{1} d^{2}+b_{2} d^{2} h+b_{3} d h^{2}+b_{4} h^{2}$ & Naslund \\
04 & $v=b_{o}+b_{1} d+b_{2} d^{2}+b_{3} d h+b_{4} d^{2} h+b_{5} h$ & Meyer \\
05 & $v=b_{o}+b_{1} d+b_{2} d^{2}+b_{3} d h+b_{4} d^{2} h$ & Meyer modificada \\
06 & $\ln v=b_{o}+b_{1} \ln d^{2} h$ & Spurr logaritmizada \\
07 & $\ln v=b_{o}+b_{1} \ln d+b_{2} \ln h$ & Schumacher-Hall \\
08 & $\ln v=b_{o}+b_{1} \ln d+b_{2} \ln ^{2} d+b_{3} \ln h+b_{4} \ln ^{2} h$ & Baden-Wurt \\
09 & $v=b_{0}+b_{1} d^{2}+b_{2} h+b_{3} d^{2} h$ & \\
10 & $v=b_{0}+b_{1} d^{2}+b_{2} d^{3}+b_{3} h+b_{4} \frac{1}{h}$ & Stoate \\
\hline \hline
\end{tabular}

Fonte: Finger (1992); Sendo: $v=$ volume comercial com casca $\left(m^{3}\right) ; d=$ diâmetro a altura do peito $(c m)$; $h=$ altura total $(m) b_{o^{\prime}} b_{1}, b_{2}, b_{3^{\prime}} b_{4}$ e $b_{5}=$ coeficientes.

\section{Equações de volume}

Para estudar o crescimento volumétrico em função do DAP e a altura total foram ajustadas 9 equações de simples entrada e 10 de dupla entrada, conforme é apresentado nas Tabelas 3 e 4 .

\section{Fator de forma comercial}

O comportamento do fator de forma comercial em função das variáveis DAP e altura, foi estudado mediante o ajuste das equações apresentadas na Tabela 5. Observa-se nesta Tabela, que algumas equações exigiram o cálculo do diâmetro relativo a 30\% da altura total da árvore. Neste caso, este diâmetro relativo foi obtido por interpolação matemática.

\section{Seleção de equações}

Como critério de escolha para as equações de relação hipsométrica, volume e fator de forma comercial utilizou-se os parâmetros do coeficiente de determinação ajustado $\left(R^{2} a j\right)$, erro padrão de estimativa em porcentagem (Syx\%), Índice de Furnival em porcentagem (IF\%), e a análise de tendência entre os valores estimados e observados. A equação escolhida para representar o comportamento de cada variável analisada foi aquela que apresentou maiores valores de $R^{2} a j$, menores valores de Syx\% e IF\% e distribuição não tendenciosa entre os valores observados e estimados.

\section{RESULTADOS E DISCUSSÃO}

Após realizados os levantamentos obteve-se os parâmetros dendrométricos médios para as quatro espécies estudadas, que podem ser observados na Tabela 6 .

Observa-se, na Tabela 6, que as espécies analisadas apresentaram bom crescimento, destacando-se a castanha-dobrasil, que apresentou maiores valores para DAP, altura média, altura dominante, área basal e volume comercial por hectare. $\mathrm{O}$ maior valor de altura dominante observado para a castanha-dobrasil é consistente com a maior produção em volume comercial por hectare, indicando que esta espécie cresce de maneira mais vigorosa nas condições de sítio estudadas.

Analisando a área basal por hectare, observa-se que mesmo com um menor número de árvores do que o ipê-roxo e o jatobá, a castanha-do-brasil apresenta maior área basal por hectare, resultante de um maior crescimento diamétrico.

$\mathrm{O}$ fator de forma comercial indicou que a andiroba e a castanha-do-brasil apresentam troncos mais cilindrícos. Os menores valores obtidos para o jatobá, indicam que esta espécie apresenta troncos mais cônicos.

Observa-se pela análise do incremento médio anual em diâmetro, altura e volume comercial, que todas as espécies estudadas apresentaram bom desempenho, com incrementos 


\section{ACTA AMAZONICA}

DENDROMETRIA DE ESPÉCIES NATIVAS EM PLANTIOS HOMOGÊNEOS NO ESTADO DE RORAIMA ANDIROBA (Carapa guianensis AUBL), CASTANHA-DO-BRASIL (Bertholletia excelsa BONPL.), IPÊROXO (Tabebuia avellanedae LORENTZ EX GRISEB) E JATOBÁ (Hymenaea courbaril L.)

Tabela 5. Equações de fator de forma comercial testadas para Carapa guianensis (andiroba), Bertbolletia excelsa (Castanhado-brasil),Tabebuia avellanedae (ipê-roxo) e Hymenaea courbaril (jatobá).

\begin{tabular}{ll}
\hline \hline $\begin{array}{l}\text { equação } \\
01\end{array}$ & $f=b_{0}+b_{1} \frac{1}{d^{2} h}+b_{2} \frac{1}{h}+b_{3} \frac{1}{d^{2}}$ \\
02 & $f=b_{0}+b_{1} \frac{1}{d^{2} h}+b_{2} \frac{1}{d h}+b_{3} \frac{1}{d}+b_{4} \frac{1}{h}+b_{5} \frac{1}{d^{2}}$ \\
03 & $f=b_{0}+b_{1} \frac{1}{h}+b_{2} \frac{h}{d}+b_{3} \frac{h}{d^{2}}$ \\
04 & $f=b_{o}+b_{1} \frac{d_{0,3}}{d}+b_{2} \frac{h}{d^{2}}$ \\
05 & $f=b_{o}+b_{1} \frac{d_{0,3}^{2}}{d}+b_{2} \frac{h}{d^{2}}$ \\
06 & $f=b_{o}+b_{1} \frac{d_{0,3}}{d^{2}}+b_{2} \frac{h}{d^{2}}+b_{3} \frac{1}{d}$ \\
08 & $f=b_{o}+b_{1} \ln d+b_{2} \ln d_{0,3}+b_{3} \ln h$ \\
\hline \hline & $f=b_{1} \ln d+b_{2} \ln \frac{h}{d=}$
\end{tabular}

Sendo $\mathrm{f}=$ fator de forma comercial; $\mathrm{d}=$ diâmetro a altura do peito $(\mathrm{cm}) ; \mathrm{d}_{0,3}=$ diâmetro tomado a $30 \%$ da altura total da árvore $(\mathrm{cm}) ; \mathrm{h}=$ altura total $(\mathrm{m}) ; \mathrm{b}_{\mathrm{o}^{\prime}} \mathrm{b}_{1^{\prime}}$ $b_{2}, b_{3}, b_{4}$ e $b_{5}=$ coeficientes.

médios em diâmetro acima de $1 \mathrm{~cm}$. O bom crescimento de espécies nativas em plantios puros na Amazônia também foi relatado por Miranda \& Valentin (2000), que obtiveram incrementos médios anuais de 3,92 cm para Espinheiro-preto (Acacia pollypbylla), 2,37 para o taperebá (Spondias lutea) e 1,65 para o cedro (Cedrela odorata) aos 4,5 anos de idade.

Barbosa et al. (2003), ao analisar o crescimento diamétrico de plantios puros e mistos de jatobá (Hymenaea courbaril), com o pará-pará (Jacaranda copaia) e pau-de-balsa (Ochroma lagopus), obtiveram diâmetros de 1,14;0,98; 1,23 cm respectivamente, aos dois anos de idade.

Os altos valores de incremento médio anual em diâmetro, que variaram entre $1,2 \mathrm{~cm}$ para o jatobá e $1,9 \mathrm{~cm}$ para a castanhado-brasil (Tabela 6), mostram-se bem superiores aos observados em florestas naturais na Amazônia. Silva et al. (1995), observaram um incremento periódico anual em diâmetro de $0,3 \mathrm{~cm}$ para todas as espécies e $0,4 \mathrm{~cm}$ para as espécies comerciais, onze anos após a colheita. No entanto, Uhl (1987) menciona incrementos de $1,8 \mathrm{~cm} /$ ano para espécies pioneiras como Vismiasp.

\section{Relação hipsométrica}

O ajuste das equações de relação hipsométrica selecionadas por espécie, bem como os parâmetros estatísticos utilizados para a seleção de equações, é apresentado na Tabela 7. A Figura 1 mostra os valores observados e ajustados, para cada espécie, e a Figura 2, a comparação entre curvas diâmetro/altura entre espécies.

A análise do coeficiente de determinação ajustado, erro padrão de estimativa em porcentagem e análise das tendências entre os valores observados e estimados, indicaram a equação 15 (equação de Prodan) como a de melhor ajuste para as quatro espécies analisadas.

Este resultado indica que uma mesma equação pode ser utilizada, independente da espécie, sendo necessário somente o ajuste em separado. Este resultado, de certa forma, concorda com Silva (1978) ao verificar que o agrupamento de canelas (Nectandra sp.) não prejudicou a estimativa das alturas. No entanto, afirmou que a diferenciação entre espécies poderia ser desprezada em seu estudo biométrico.

Observa-se na Figura 2, que as espécies apresentaram comportamento distinto em relação à curva de relação hipsométrica, indicando a necessidade de se fazer o ajuste em separado por espécie. Este resultado concorda com Loetsch et al. (1973) ao afirmar que a relação altura/diâmetro varia com a espécie, sendo o ângulo de curvatura característico de cada espécie.

A castanha-do-brasil apresentou maior inclinação atingindo maiores alturas nos maiores diâmetros. Nos menores diâmetros observa-se superioridade para o ipê-roxo.
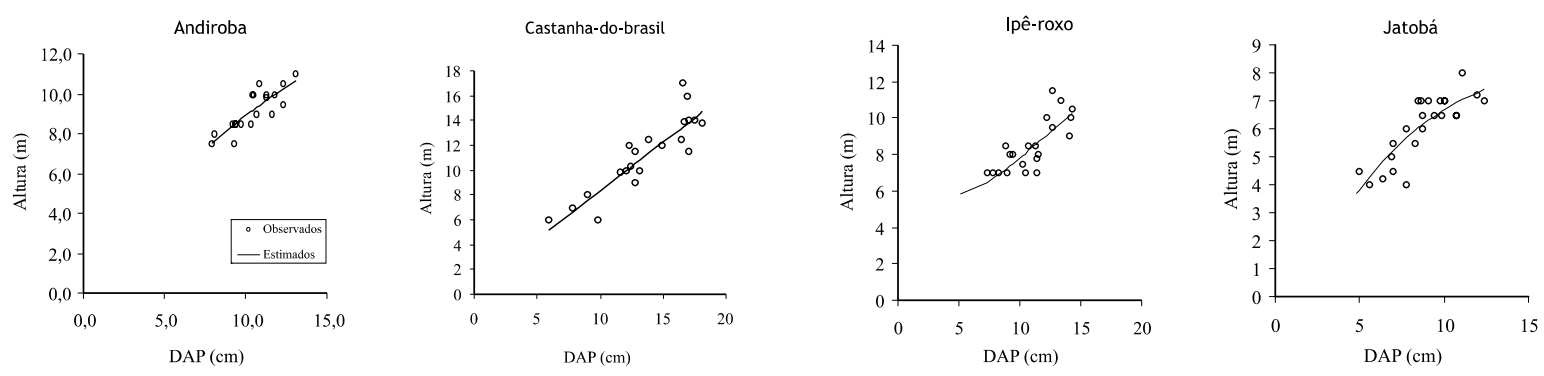

Figura 1 - Valores observados e estimados para a curva altura/diâmetro nas espécies analisadas. 


\section{ACTA AMAZONICA}

DENDROMETRIA DE ESPÉCIES NATIVAS EM PLANTIOS HOMOGÊNEOS NO ESTADO DE RORAIMA ANDIROBA (Carapa guianensis AUBL), CASTANHA-DO-BRASIL (Bertholletia excelsa BONPL.), IPÊROXO (Tabebuia avellanedae LORENTZ EX GRISEB) E JATOBÁ (Hymenaea courbaril L.)

\section{Volume comercial}

O ajuste das equações de volume de simples e dupla entrada, selecionadas por espécie, bem como os parâmetros estatísticos utilizados para a seleção de equações são apresentados na Tabela 8. A Figura 3 mostra o ajuste para as equações volumétricas e a Figura 4 , a comparação entre espécies.

Observa-se, na Tabela 8, melhor ajuste para as equações de dupla entrada que apresentaram os menores valores de erro padrão de estimativa em porcentagem (Syx\%). Este resultado se encontra dentro do esperado, uma vez que a altura responde por grande parte da variação em volume de uma árvore. Autores como Silva \& Araujo (1984) e Silva \& Carvalho (1984), também obtiveram maior precisão em equações de dupla entrada para espécies nativas em florestas naturais.

No entanto, a superioridade para as equações de dupla entrada pode ser considerada pequena, como pode ser constatado pelos valores de $\mathrm{R}^{2}$ aj na Tabela 8 . A utilização de equações de simples entrada pressupõe que árvores de uma mesma classe diamétrica tenham similar altura $\mathrm{e}$ forma, o que na maioria dos casos não ocorre. A pouca diferença entre as equações de volume de simples e dupla entrada pode ser explicada pela pequena variação em altura observada nas parcelas e pelas dimensões reduzidas das mesmas, diminuindo a possibilidade de existirem variações de sítio.

Na Tabela 8, observa-se que tanto para as equações de simples entrada como para as de dupla entrada não foi possível utilizar a mesma equação independente da espécie, pois a equação de melhor ajuste variou com as mesmas. Este resultado concorda com Souza \& Jesus (1991), que também verificaram diferenças entre espécies nas equações testadas para volume comercial em três espécies nativas em florestas naturais.

A equação volumétrica de dupla entrada de melhor ajuste para a andiroba, castanha-do-brasil, ipê-roxo e jatobá foram as de números 2 (Stoate), 1 (Spurr), 4 (Meyer) e 10 (Stoate), respectivamente.

O ajuste de diferentes equações para diversas espécies, pode ser desejável na medida em que o volume de uma árvore pode ser estimado através do DAP, altura, $\mathrm{e}$ às vezes, uma medida de forma do tronco. No entanto, a forma do tronco é uma variável de difícil obtenção, ocorrendo ainda variações intra e inter específicas.
Embora a forma do tronco não seja explicitamente incluída como variável independente em uma equação de volume, estas diferenças tendem a ser levadas em consideração ajustando-se equações separadas por espécie ou grupos de espécies.

Observa-se, na Figura 4, grande superioridade no crescimento volumétrico da castanha-do-brasil e da andiroba em relação ao ipê-roxo e ao jatobá. Esta superioridade deve-se a um maior crescimento em altura e diâmetro (Tabela 6) e ao comprimento do fuste, que no ipê-roxo e jatobá, mostraramse curtos com cerca de 2 metros.

\section{Fator de forma}

O ajuste das equações de fator de forma comercial, selecionadas por espécie, bem como os parâmetros estatísticos utilizados para a seleção de equações, são apresentados na Tabela 9. A Figura 5 mostra o ajuste para as equações selecionadas e a Figura 6 , a comparação entre espécies.

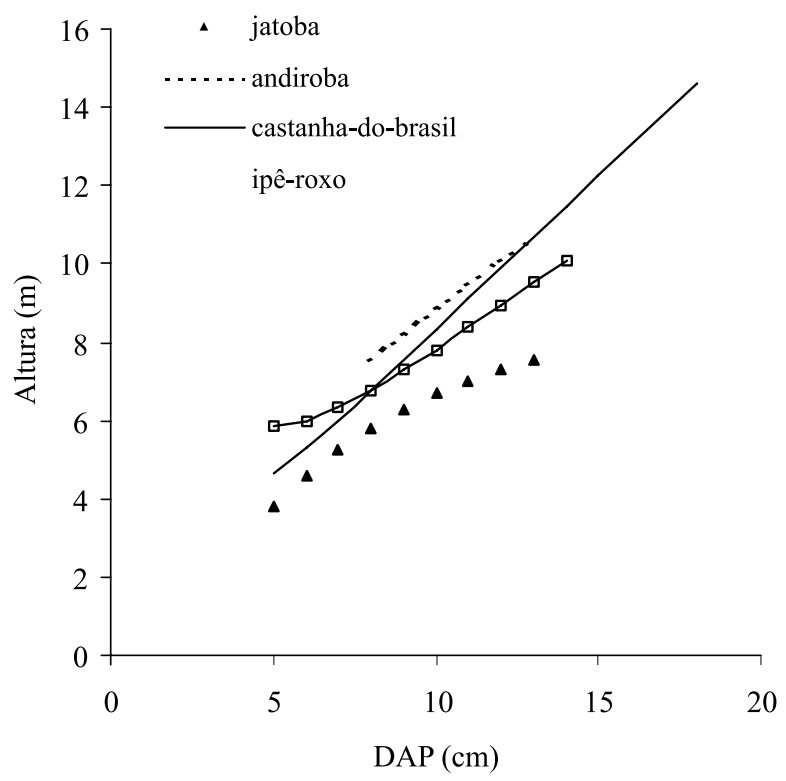

Figura 2 - Comparações entre as curvas de altura/diâmetro para as espécies analisadas.

Tabela 6 - Parâmetros dendrométricos médios para as espécies estudadas, aos 7 anos de idade.

\begin{tabular}{|c|c|c|c|c|c|c|c|c|c|c|}
\hline Espécie & $\mathrm{N}$ & $\begin{array}{l}\mathrm{d} \\
(\mathrm{cm})\end{array}$ & $\begin{array}{l}\mathrm{h} \\
(\mathrm{m})\end{array}$ & $\begin{array}{l}\mathrm{h}_{100} \\
(\mathrm{~m})\end{array}$ & $\mathrm{f}$ & $\begin{array}{l}\text { G. } \\
\left(\mathrm{m}^{2} / \mathrm{ha}\right)\end{array}$ & $\begin{array}{l}\text { V } \\
\left(\mathrm{m}^{3} / \mathrm{ha}\right)\end{array}$ & $\begin{array}{l}\text { IMAd } \\
(\mathrm{cm})\end{array}$ & $\begin{array}{l}\text { IMAh } \\
\text { (m) }\end{array}$ & $\begin{array}{l}\text { IMAV } \\
\left(\mathrm{m}^{3}\right)\end{array}$ \\
\hline Andiroba & 1055 & 10,5 & 9,2 & 10,7 & 0,50 & 9,3 & 43,8 & 1,5 & 1,3 & 6,3 \\
\hline Castanha-do-brasil & 1167 & 13,5 & 11,3 & 13,9 & 0,48 & 17,8 & 102,5 & 1,9 & 1,6 & 14,6 \\
\hline Ipê-roxo & 1222 & 10,7 & 8,3 & 10,2 & 0,44 & 11,5 & 42,3 & 1,5 & 1,2 & 6,0 \\
\hline Jatobá & 1389 & 8,6 & 6,0 & 7,1 & 0,38 & 8,6 & 20,6 & 1,2 & 0,8 & 2,9 \\
\hline
\end{tabular}

Sendo: $\mathrm{N}=$ número de árvores por hectare; $\mathrm{d}=$ diâmetro a $1,30 \mathrm{~m}$ do solo; $\mathrm{h}=$ altura total; $\mathrm{h}_{100}=$ altura dominante (definida segundo o conceito de Assmann); $\mathrm{f}=$ fator de forma comercial; $\mathrm{G}=$ área basal por hectare; $\mathrm{V}=$ volume comercial por hectare; $I M \mathrm{~A}_{\mathrm{d}}=$ incremento médio anual em diâmetro; $I \mathrm{MA}=$ incremento médio anual em altura; $I M A_{v}=$ incremento médio anual em volume comercial. 


\section{ACTA \\ AMAZONICA}

DENDROMETRIA DE ESPÉCIES NATIVAS EM PLANTIOS HOMOGÊNEOS NO ESTADO DE RORAIMA ANDIROBA (Carapa guianensis AUBL), CASTANHA-DO-BRASIL (Bertholletia excelsa BONPL.), IPÊROXO (Tabebuia avellanedae LORENTZ EX GRISEB) E JATOBÁ (Hymenaea courbaril L.)

Tabela 7. Ajuste para as equações de relação hipsométrica testadas para Carapa guianensis (andiroba), Bertholletia excelsa (castanha-do-brasil), Tabebuia avellanedae (ipê-roxo) e Hymenaea courbaril (jatobá).

\begin{tabular}{llllll}
\hline \hline & \multicolumn{2}{l}{ Coeficientes } & \multicolumn{2}{l}{ Estatísticas } \\
\cline { 2 - 6 } Espécie & $\mathrm{b} 0$ & $\mathrm{~b} 1$ & $\mathrm{~b} 2$ & $\mathrm{R}^{2} \mathrm{aj}$ & $\mathrm{Syx} \%$ \\
\hline Andiroba & $-1,1888$ & 1,7502 & 0,1999 & 0,97 & 3,81 \\
Castanha- & $-4,3675$ & 3,0076 & 0,1206 & 0,95 & 4,53 \\
do-brasil & & & & & \\
Ipê-roxo & $-8,8722$ & 3,4217 & 0,1382 & 0,96 & 6,89 \\
Jatobá & 5,1594 & 0,4533 & 0,3344 & 0,96 & 7,20 \\
\hline \hline
\end{tabular}

Sendo: $b_{0}, b_{1}, b_{2}=$ coeficientes; $R^{2} a j=$ coeficiente de terminação ajustado; Syx $\%$ $=$ Erro padrão de estimativa em porcentagem.
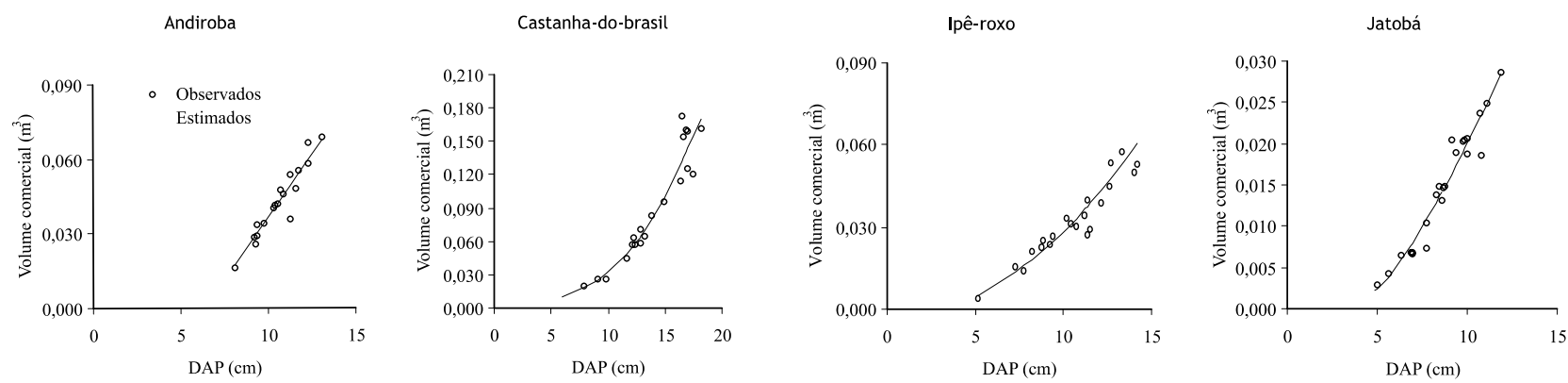

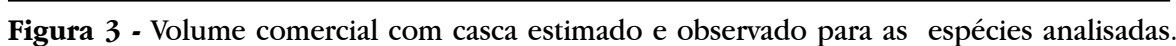

Tabela 8 - Ajuste das equações de volume de simples e dupla entrada para as espécies analisadas.

\begin{tabular}{|c|c|c|c|c|c|c|c|c|c|c|}
\hline \multirow[b]{2}{*}{ Espécie } & \multirow[b]{2}{*}{$\mathrm{N}^{0}$} & \multicolumn{6}{|c|}{ Coeficientes } & \multicolumn{3}{|c|}{ Estatísticas } \\
\hline & & $\mathrm{b}_{0}$ & $b_{1}$ & $b_{2}$ & $b_{3}$ & $b_{4}$ & $b_{5}$ & $R^{2} a j$ & Syx\% & IF \% \\
\hline andiroba ${ }^{(1)}$ & 01 & $-0,0647$ & 0,0101 & & & & & 0,91 & 10,6 & \\
\hline cast. -do-brasil(1) & 09 & $-6,7171$ & 0,3995 & $-0,0069$ & & & & 0,97 & & 23,7 \\
\hline ipê-roxo(1) & 02 & $-0,0031$ & 0,0003 & & & & & 0,95 & 19,3 & \\
\hline jatobá(1) & 07 & $-0,0399$ & 0,0826 & 0,0052 & & & & 0,95 & 11,8 & \\
\hline andiroba ${ }^{(2)}$ & 02 & $-0,0212$ & 0,0004 & $2,65^{*} 10^{-6}$ & 0,0016 & & & 0,91 & 10,9 & \\
\hline cast. -do-brasil(2) & 01 & 0,0038 & $3,47^{*} 10^{-5}$ & & & & & 0,97 & 10,7 & \\
\hline ipê-roxo ${ }^{(2)}$ & 04 & $-0,2114$ & 0,0639 & $-0,0037$ & $-0,0062$ & $4^{*} 10^{-4}$ & 0,017 & 0,91 & 14,9 & \\
\hline jatobá(2) & 10 & $-0,0317$ & 0,0003 & $-6,1^{*} 10^{-6}$ & 0,0028 & $666 * 10^{-4}$ & & 0,95 & 11,7 & \\
\hline
\end{tabular}

Sendo: (1) = equação de simples entrada; ( 2 ) = equação de dupla entrada; $N^{\circ}=$ número da equação; $b_{0^{\prime}} b_{1^{\prime}}, b_{2}, b_{3}, b_{4}$ e $b_{5}=$ coeficientes; $R^{2} a j=$ coeficiente de determinação ajustado; Syx $\%$ = Erro padrão de estimativa em porcentagem. 


\section{ACTA AMAZONICA}

DENDROMETRIA DE ESPÉCIES NATIVAS EM PLANTIOS HOMOGÊNEOS NO ESTADO DE RORAIMA ANDIROBA (Carapa guianensis AUBL), CASTANHA-DO-BRASIL (Bertholletia excelsa BONPL.), IPÊROXO (Tabebuia avellanedae LORENTZ EX GRISEB) E JATOBÁ (Hymenaea courbaril L.)

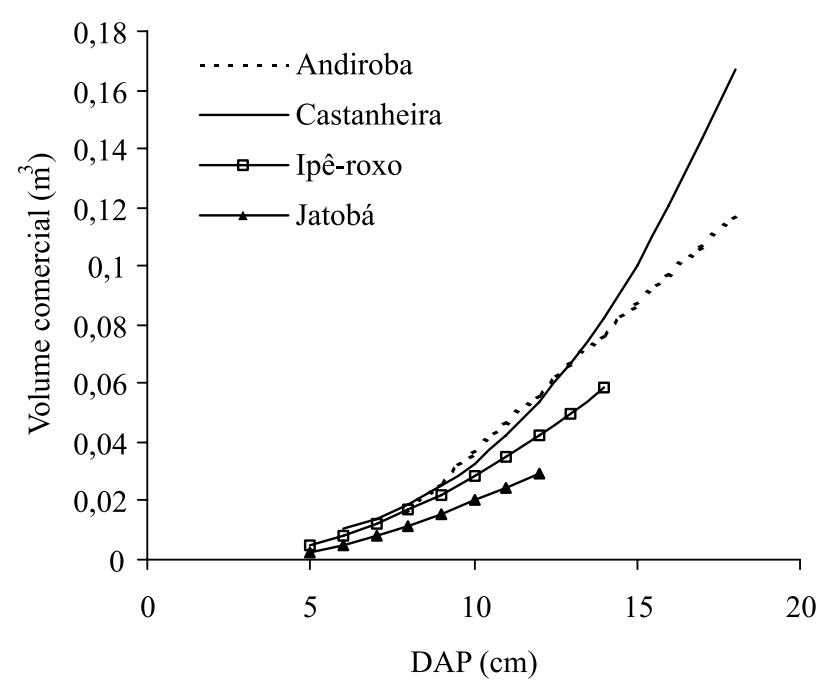

Figura 4 - Curvas de volume comercial com casca em função do DAP para as espécies analisadas.

A Figura 6 mostra que, exceto a andiroba, as demais espécies apresentam a tendência de diminuição do fator de forma comercial, conseqüentemente tornaram-se mais cônicas, com o aumento do diâmetro. A andiroba mostrou tendência inversa para árvores de menores dimensões, porém, a partir dos $10 \mathrm{~cm}$ de diâmetro, também apresentou tendência decrescente.

Para o jatobá, a equação selecionada (6), tem como variável independente o diâmetro a $30 \% \mathrm{da}$ altura da árvore $\left(\mathrm{d}_{0,3}\right)$, sendo necessário, por motivos práticos, estimá-lo em função do DAP. A Figura 7 mostra que a relação entre o d $_{03}$ e o DAP é linear, sendo estimada pela equação da reta. Esta equação apresentou intercepto igual $\mathrm{a}-0,2785$, coeficiente angular igual a 0,9955 e valores de $R^{2}$ aj e Syx de 0,92 e 0,43, respectivamente.

\section{CONCLUSÕES}

Após a realização deste trabalho pode-se concluir que:

a) As espécies analisadas apresentaram bom crescimento nas condições de sitio estudadas, mostrando-se promissoras tanto para o uso em reflorestamentos como em sistemas agroflorestais no estado de Roraima. Das espécies estudadas, destacou-se a castanha-do-brasil (Bertholletia excelsa) com maiores valores de incremento médio anual em diâmetro e volume comercial.

b) A equação hipsométrica de Prodan, apresentou melhor ajuste para todas as espécies. No entanto, a análise gráfica indicou que a forma da curva altura/diâmetro variou com a espécie, sendo necessário o ajuste em separado.

c) As equações de volume de simples e dupla entrada apresentaram ajustes semelhantes. A melhor equação variou com a espécie sendo selecionadas as equações de Stoate (2) para a andiroba (Carapa guianensis), a de Spurr para a castanhado-brasil (Bertholletia excelsa), a de Meyer (4) para o ipê-roxo (Tabebuia avellanedae) e a de Stoate (10) para o jatobá (Hymenaea courbaril).

d) A análise do fator de forma comercial indicou que, com exceção da andiroba, o mesmo tende a diminuir com o aumento do DAP. As espécies analisadas apresentaram um fator
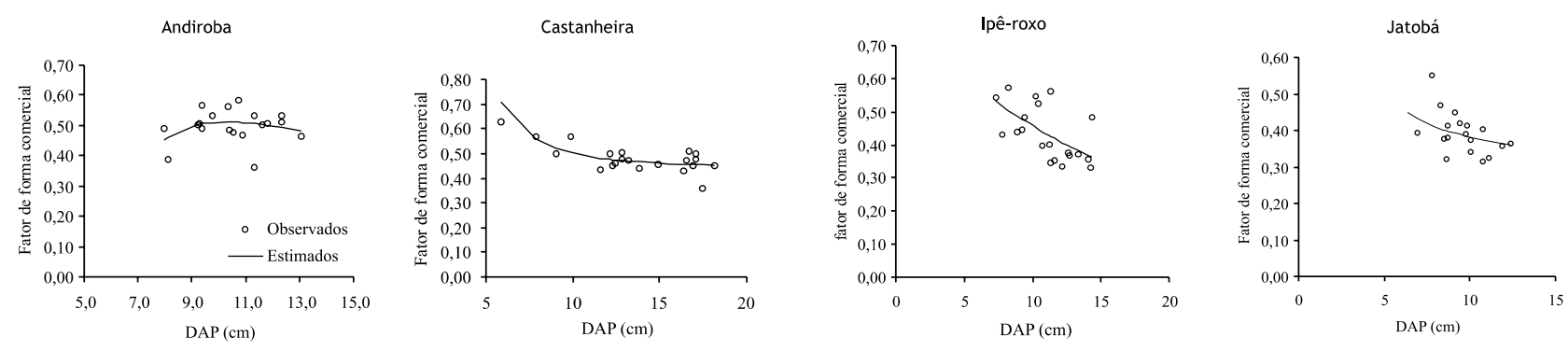

Figura 5 - Valores observados e ajustados para o fator de forma comercial nas espécies analisadas.

Tabela 9 - Ajuste das equações de fator de forma comercial para as espécies analisadas.

\begin{tabular}{|c|c|c|c|c|c|c|c|}
\hline \multirow[b]{2}{*}{ Espécie } & \multirow[b]{2}{*}{$\mathrm{N}^{0}$} & \multicolumn{3}{|c|}{ Coeficientes } & \multicolumn{3}{|c|}{ Estatísticas } \\
\hline & & $\mathrm{b}_{0}$ & $b_{1}$ & $\mathrm{~b}_{2}$ & $b_{3}$ & $R^{2} a j$ & Syx\% \\
\hline Andiroba & 01 & $-0,0927$ & $-363,2590$ & 6,5393 & 27,6772 & 0,09 & 10,41 \\
\hline Castanha-do-brasil & 01 & 0,4202 & 77,4569 & 0,6573 & $-9,2490$ & 0,62 & 7,27 \\
\hline Ipê-roxo & 10 & 1,0867 & $-0,4577$ & 0,2695 & $-0,4804$ & 0,49 & 16,11 \\
\hline Jatobá & 06 & 0,1755 & 0,6363 & $-3,3697$ & 3,6973 & 0,61 & 10,87 \\
\hline
\end{tabular}

Sendo: $\mathrm{N}^{\mathrm{o}}=$ número da equação; $\mathrm{b}_{0^{\prime}} \mathrm{b}_{1}, \mathrm{~b}_{2}, \mathrm{~b}_{3}=$ coeficientes; $\mathrm{R}^{2} \mathrm{aj}=$ coeficiente de determinação ajustado; Syx $\%=$ Erro padrão de estimativa em porcentagem. 


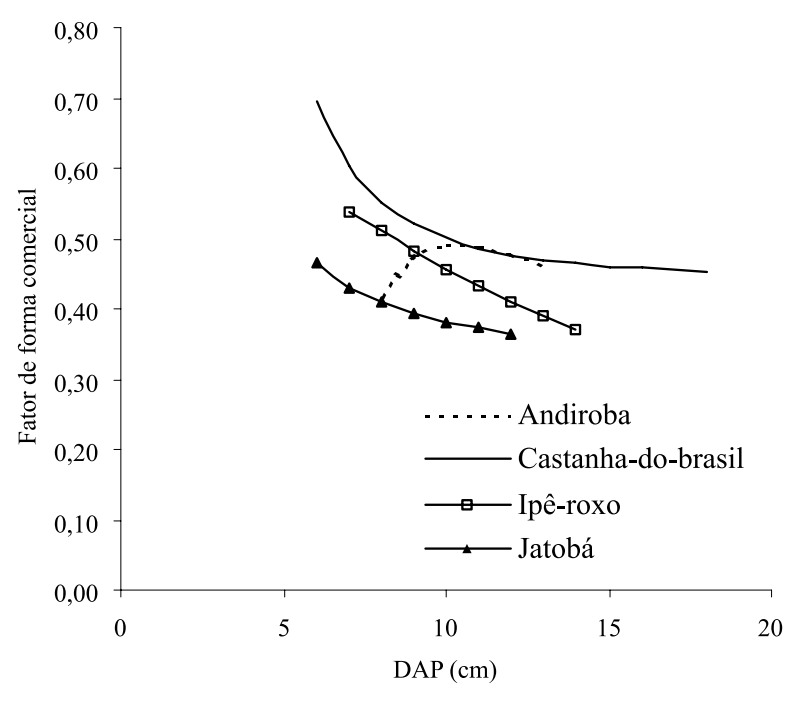

Figura 6 - Curvas para o fator de forma comercial em função do DAP, para as espécies analisadas.

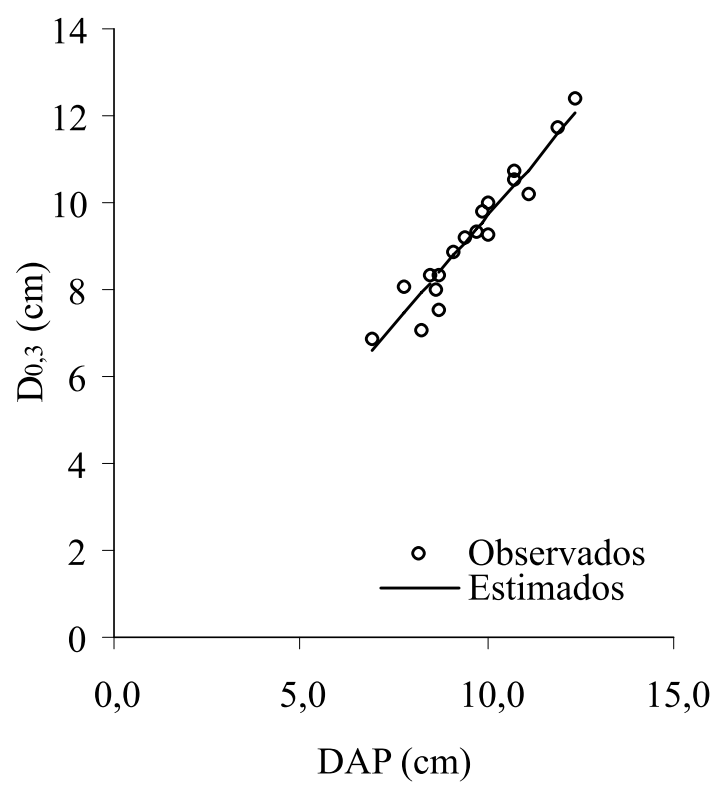

Figura 7 - Valores observados e estimados para o diâmetro a $30 \%$ da altura da árvore para o jatobá (Hymenaea courbaril).

de forma comercial distinto, indicando que a utilização de um fator de forma médio para todas as espécies deve ser evitado, como forma de diminuir os erros nas estimativas do volume comercial.

\section{BIBLIOGRAFIA CITADA}

Andrae F. 1978. Ecologia florestal. Ed. da UFSM, Santa Maria, $100 \mathrm{p}$.

Barbosa, A. P.; Iida, S.;Vieira, G.; Sampaio P.T.B.; Oliveira, L.A..; Campos, M.A.A.;Pinto, A.M.; Spironello W.R.; Gonçalves, C.B.Q.; Almeida, M.J.B.; Neves, T.S.; Cortes A.M.L. 2003. Silvicultura tropical e a recuperação de áreas degradadas pela agricultura etinerante na Amazônia Central. In: Higuch, N., Santos, J., Sampaio, P.T.B et al.Projeto jacaranda Fase II: Pesquisas florestais na Amazônia Central. Instituto Nacional de Pesquisas da Amazônia (INPA). Manaus, Amazonas, p.223-239.

EMBRAPA. 1999. Sistema brasileiro de classificação de solos. Embrapa Solos, Rio de Janeiro, 412 p.

Ferreira, M. 1987. Escolha de espécies arbóreas para a formação de maciços. Documentos florestais. ESALQ. Piracicaba, 15 p.

Lamprecht, H. 2000. Silvicultura nos trópicos: ecossistemas florestais e respectivas espécies arbóreas - possibilidades $e$ métodos de aproveitamento sustentado. Dt.Ges.für Techn.Zusammenarbeit, Rossdorf, República Federal da Alemanha. 343 p.

Loestch, F.; Zohrer, F.; Haller, K.E. 1973. Forest Inventory, BLY Verlagsgesellschaft: München, República Federal da Alemanha. $150 \mathrm{p}$.

Miranda, E.M.; Valentim, J.F. 2000. Desempenho de doze espécies arbóreas nativas e introduzidas com potencial de uso múltiplo no Estado do Acre, Brasil. Acta Amazonica 30 (3): 471-480.

Prodan, M.; Peters, R.; Cox, F.; Real, P. 1997. Mensura Forestal. (GTZ) Gmbh/IICA,Costa Rica, 561 p.

Silva, J.A.1978. Comprimento do tronco, relação altura-diâmetro e altura-idade do pinheiro brasileiro (Araucaria angustifolia (Bert.) O.Ktze). e folhosas associadas em ambiente natural. Revista Brasil Florestal, 34: 43-50.

Silva, J.N.M .; Carvalho, M.S.P. 1984. Equações de volume para uma floresta secundária no Planalto do Tapajós Belterra, PA. Boletim de Pesquisa Florestal, 8 (9), Embrapa CPATU, p. $1-15$.

Silva, J.N.M .; Araújo, S.M. 1984. Equação de volume para árvores de pequeno diâmetro, na Floresta Nacional do Tapajós.Boletim de Pesquisa Florestal, 8 (9), Embrapa CPATU. p.17-25.

Silva, J.N.M.; Carvalho, J.O.P; Lopes, J.C.A..; Almeida, B.F.; Costa D.H.M.; Oliveira, L.C.; Vanclay, J.K.; Skovsgaard, J.P. 1995. Growth and yield of a tropical rain forest in the Brazilian Amazon 13 years after logging. Forest Ecology and Management, 71: 267-274.

Souza, A.L.; Jesus, R.M. 1991. Equações de volume comercial e fator de forma para espécies da mata atlantica ocorrentes na reserva florestal da Companhia Vale do Rio Doce, Linhares, ES. Revista Árvore 15(3):257-273. 
DENDROMETRIA DE ESPÉCIES NATIVAS EM PLANTIOS HOMOGÊNEOS NO ESTADO DE RORAIMA ANDIROBA (Carapa guianensis AUBL), CASTANHA-DO-BRASIL (Bertholletia exce/sa BONPL.), IPÊROXO (Tabebuia avellanedae LORENTZ EX GRISEB) E JATOBÁ (Hymenaea courbaril L.)

Thibau, C.E. 2000. Produção sustentada em florestas: Conceitos e tecnologias, biomassa energética, pesquisas e constatações. Ed. do autor, Belo Horizonte. 509 p.

Uhl. C. 1987. Factors controlling succesion following slashand-burn agriculture in Amazonia. Journal of Ecology, 75: 377-407.
Wadsworth, F. H. 2000. Producción forestal para América Tropical. USDA, Washington, USA, $602 \mathrm{p}$.

RECEBIDO EM 04/09/2003

ACEITO EM 21/09/2005 